\title{
Philosophiques
}

\section{La critique piagétienne de l'apriorisme attaque-t-elle le criticisme kantien?}

\section{Maurice Gagnon}

Volume 7, numéro 1, avril 1980

URI : https://id.erudit.org/iderudit/203130ar

DOI : https://doi.org/10.7202/203130ar

Aller au sommaire du numéro

Éditeur(s)

Société de philosophie du Québec

ISSN

0316-2923 (imprimé)

1492-1391 (numérique)

Découvrir la revue

Citer cet article

Gagnon, M. (1980). La critique piagétienne de l'apriorisme attaque-t-elle le criticisme kantien? Philosophiques, 7(1), 41-54. https://doi.org/10.7202/203130ar d'utilisation que vous pouvez consulter en ligne.

https://apropos.erudit.org/fr/usagers/politique-dutilisation/ 


\title{
LA CRITIQUE PIAGÉTIENNE DE L'APRIORISME ATTAQUE-T-ELLE LE CRITICISME KANTIEN ?
}

\author{
par Maurice Gagnon
}

\section{PIAGET ET KANT : UNE OPPOSITION POSSIBLE}

Le présent texte a pour but de déterminer s'il faut voir dans les critiques piagétiennes de l'apriorisme une réfutation, voulue ou non par Piaget, du criticisme kantien. Il est plutôt rare que Piaget parle de Kant dans ses écrits. Et quand il le fait, c'est plutôt sous la forme d'une mention rapide'. Mais certaines des descriptions et critiques de l'apriorisme semblent bien viser, entre autres, la philosophie kantienne:

Un troisième point de vue, celui de l'apriorisme pur, considérerait l'espace comme inné parce que constituant le mode

1. Voici les quelques allusions explicites à Kant que nous avons trouvées dans les écrits de Piaget. Dans NI p. 328-239: «... déçu tout à la fois par l'empirisme anglais et par l'intellectualisme classique (et surtout par la thérie wolffienne de la faculté rationnelle), le kantisme recourut à l'hypothèse aprioriste pour explicuer la possibilité de la science. ”

Dans EG, tome I, p. 148, Piaget classe l'espace de Kant parmi les théories non génétiques de l'espace, soulignant que l'espace kantien est le sensorium bominis alors que celui de Newton est le sensorium Dei. À la page suivante, il est dit que l'espace de Kant "se fonde sur une élaboration endogène", et que l'apriorisme kantien est " la traduction psychologique et physiologique " d'un "nativisme " qui « réduit la perception de l'espace à une conscience de notre propre organisation, cette dernière assimilant à sa structure interne les données externes $»$. Voir aussi BC, p. 95, 310.

Les ouvres de Piaget seront, dans cet article, désignées par les signes suivants, que nous empruntons à Antonio M. Battro dans Dictionnaire d'épistémologie génétique, P.U.F., 1966 :

BC Biologie et connaissance, Gallimard, 1967

CR La construction du réel chez l'enjant, 4cédition, Delachaux et Niestlé, 1967.

DQ Le développement des quantités ṕnysiques dbez l'enfant, $2^{c}$ édition, Delachaux et Niestlé, 1962.

EG Introduction à l'épistémologie générique, P.U.F., 1950.

E.E.G. XIV, Études d'épistémologie génétique, vol. XIV, Épistémologie mathématique et psycholngie. Essai sur les relations entre la logique formelle et la pensée réelle (avec E. W. Beth), P.U.F., 1961.

JM Le jugement moral thez l'enfant, P.U.F., 1957.

NI La natsance de l'intelligence chez l'enfant, $5^{e}$ édition, Delachaux et Niestlé, 1967.

PE Psychologie et épistémolugie, Gontheir, 1970.

PI La psychologie de l'intelligence, Armand Colin, 1947.

RE La représentation de l'espate chez l'enfant (avec B. Inhelder) P.U.F., 1948. 
nécessaire de perception propre aux organes des sens: la connaissance spatiale ne devrait donc rien au milieu, l'espace étant simplement projeté par l'esprit dans les choses sans leur appartenir en soi $^{2}$.

En troisième lieu, l'interprétation aprioriste de la causalité implique une hypothèse psychologique, selon laquelle la notion de cause constitue une structure nécessaire inhérente à tout acte intellectuel et donnée, par conséquent, dès les premiers contacts de l'esprit avec la réalité $e^{3}$.

. . au préformisme correspond l'apriorisme qui explique la connaissance par des structures internes antérieures à l'expérience ${ }^{4}$.

On ne peut s'empêcher, en lisant ces lignes, de penser à Kant, quand on sait que pour ce dernier l'espace et le temps sont les formes pures (c'est-à-dire ne devant rien à l'espérience) et a priori (c'est-à-dire conditions indispensables de toute expérience et par conséquent antérieures à elle), le premier de l'imagination, et le second de la mémoire. La même situation existe aussi, selon Kant, pour les notions de substance et de cause, et aussi pour le principe de causalité par rapport aux phénomènes, c'est-à-dire au résultat de l'application des formes pures et a priori espace et temps à la sensation. La notion de cause est elle aussi pure et a priori, et elle organise les phénomènes selon la loi stricte de dépendance de l'effet par rapport à la cause. La notion de substance crée une permanence d'identité sous-jacente aux phénomènes changeants.

Kant semble aussi visé à travers les critiques adressées par Piaget à la Denkpsychologie et à la psychologie gestaltiste. Sans discuter ici du bien fondé de ces critiques, ce qui nécessiterait une étude beaucoup plus longue et plus poussée, résumons-en l'essentiel. La Denkpsychologie soutient, selon Piaget, que la logique est innée à l'esprit, qui ne l'utilise et n'en prend conscience que progressivement. Piaget lui oppose les recherches dont le résultat est exposé surtout dans La genèse des structures logiques élémentaires (avec B. Inhelder, Delachaux et Niestlé, 1959), De la logique de l'enfant à la logique de l'adolescent (avec B. Inhelder, $2^{\mathrm{e}}$ édition, P.U.F., 1955), et Le jugement et 
Le raisonnement chez l'enfant, $6^{\mathrm{e}}$ édition, Delachaux et Niestlé, 1967). Les structures et lois logiques ne sont pas innées, dit-il, mais progressivement construites. Celles de la pensée enfantine ne sont pas les mêmes que celles de la pensée adulte, mais elles évoluent selon des phases successives jusqu'au stade adulte. D'ailleurs, si les structures logiques de la pensée adulte étaient innées, on ne voit pas pourquoi le sujet ne les utiliserait pas spontanément dès le début de sa vie, dès que lui est fourni un contenu de connaissance auquel elles peuvent s'appliquer.

Il semble bien que pour la Denkpsychologie, les structures logiques sont pures et a priori, comme l'espace et le temps de Kant, et aussi comme les concepts de l'entendement (v.g. cause, substance, etc., ) également chers à Kant.

Piaget est d'accord avec les gestaltistes pour admettre qu'il n'y a pas de sensations isolées et antérieures à la perception et que cette dernière n'est pas une simple synthèse ou juxtaposition de sensations, mais qu'elle est déterminée par une forme ou structure globale qui transcende les éléments et joue un rôle causal et intégrateur à leur endroit. Il leur reproche cependant de ne pas s'interroger sur la genèse de cette structure, d'admettre trop facilement son caractère pur et a priori au sens kantien de ces termes. Piaget admet également que l'établissement d'une telle structure sur un groupe d'éléments obéit à des lois de simplicité, de symétrie, d'harmonie, etc., qui sont en fait pour lui des lois d'équilibre et qui s'appliquent spontanément et automatiquement, sans calcul, sans raisonnement ou effort intellectuel volontaire, conscient et dirigé. Mais l'équilibre des contenus perceptifs, note Piaget, est rigide, alors que celui des systèmes intellectuels est souple et mobile parce que réversible, soit par inversion (négation) ou réciprocité. Les gestaltistes ont raison de dire que les systèmes intellectuels sont aussi munis d'une structure totale ou globale, dit Piaget, mais la souplesse de l'équilibre de ces systèmes en fait plus que des gestalten ${ }^{5}$.

5. Sur la cririque des psychologies de la forme et de la pensée, voir notamment NI, p. 328-345 ; PI, p. 28-34 et 64-95 ; EG, tome I, p. 165-174. 
II. LES CONCEPTS DE SUBSTANCE, D'ESPACE, DE CAUSE ET DE TEMPS CHEZ PIAGET ET KANT.

De fait, quatre notions sont également importantes pour Piaget et pour Kant, et les deux ont analysé ces notions. Ce sont les notions d'espace, de temps, de cause et d'objet. Il faut préciser ici que pour Piaget - et nous croyons qu'il a raison sur ce point - la notion de substance est un prolongement sophistiqué de la notion d'objet permanent, avec cette différence que la permanence et l'identité de l'objet reposent sur la permanence de la forme géométrique et de la dimension, alors que celles de la substance reposent sur la permanence qualitative du support de ces caractères variables ${ }^{6}$. Ce support est nécessairement quantifié pour permettre la conservation de cette qualité malgré les changements de forme et de dimensions, ce qui fait que la substance est aussi la quantité en général ${ }^{7}$.

Si on compare ce que Piaget dit de ces notions dans $L a$ construction $d u$ réel chez l'enfant et ce qu'en dit Kant dans la Critique de la Raison Pure, on constate que ce qui est pour Piaget le point d'arrivée, le résultat du processus de construction des notions chez l'enfant de deux ans, constitue au moins en partie le point de départ de la réflexion kantienne ${ }^{8}$. Voyons un peu ce qui en est pour chacune des notions mentionnées.

Au sixième stade de l'intelligence sensori-motrice, l'enfant a construit la notion diobjet permanent, dont l'existence est indépendante de sa perception, et dont il peut reconstituer les déplacements invisibles après sa disparition derrière un autre objet qui le cache. Or, la notion kantienne de substance a justement pour caractéristique spécifique la permanence dans le temps, avec cette différence qu'elle est pour Kant nécessaire, ce qui entraîne l'éternité de toute substance. Mais il est à noter que Kant utilise comme synonymes les mots «substance » et

6. DQ, p. 6-29, surtout 21-22

7. Ibid., p. 23, 24, 29, 257, 333.

8. Pour l'espace et le temps kantiens, voir dans la Critique de la Raison Pure l'Esthétique Transcendantale section 1 et II ; pour la substance et la cause, voir dans l'Analytique des Principes les deux premières analogies de l'expérience, dans la section III du chap. II. 
" matière ${ }^{9}$, ce qui s'accorde bien avec ces deux passages de Piaget :

la première signification à donner à cette notion de substance ou de matière est celle d'un schéma général de quantification, c'est-à-dire du quantum physique le plus simple et le plus indifférencié ${ }^{10}$.

... c'est l'idée que quelque chose se conserve, sans que l'enfant sache encore exactement quoi, sinon la matière comme telle c'est-à-dire une qualité indifférenciée, et par conséquent quantifiée avant toutes les autres ${ }^{11}$.

Entre 18 et 24 mois, l'enfant en arrive à organiser ses propres déplacements dans l'espace selon la structure mathématique de groupe, à prévoir les déplacements des autres personnes ou objets selon la même structure, et à coordonner de même ses propres actions dans l'espace avec celles des autres personnes ou objets. Il est en outre capable de reconstituer déductivement les déplacements non perçus des choses, et saisit la relativité des points de repère multiples. L'espace est donc décentré et objectivé par rapport au sujet qui ne se considère plus comme centre absolu et comme repère unique. Les divers espaces (buccal, visuel, tactile, etc.,) sont coordonnés en un seul. L'espace kantien est de son côté unique, objectif, uniforme et infini. Ces deux dernières caractéristiques seules manquent à l'espace de l'enfant de 2 ans, bien qu'on puisse dire que son espace est spontanément, mais non consciemment, euclidien et uniforme.

L'enfant de 2 ans recherche spontanément les causes des événements qu'il perçoit, il peut se représenter mentalement

9. Critique de la Raison Pure, trad. Tremesaygues et Pacaus, P.U.F., 1965, p. 180 : «On posait à un philosophe cette question : Combien pèse la fumée? Il répondit : retranchez du poids du bois brûlé le poids de la cendre qui reste, vous avez le poids de la fumée. Il supposait donc comme une chose incontestable que même dans le feu la matière (la substance) ne disparait pas, mais que sa forme seule subit un changement. Il en est de même de cette proposition : rien ne procède de tien; elle n'est qu'une autre conséquence du principe de la permanence, ou plutôt de l'existence toujours persistante du sujet propre des phénomènes. En effet, pour que ce qu'on veut appeler substance dans le phénomène puisse être le substrat propre de toute détermination de temps, il faut que toute existence, dans le passé comme dans le temps futur, y soit uniquement et exclusivement déterminée. »

10. DQ, p. 21 .

11. Ibid., p. 24 ; voir aussi les textes auxquels nous avons déjà renvoyé le lecteur, dans les notes 6 et 7 ci-dessus, où Piaget parle souvent de "quantité de matière ou de substance $\gg$. 
les causalités non perçues, sans pour autant formuler explicitement (ni sans doute concevoir) le principe de causalité (tout ce qui arrive a nécessairement une cause). Il est conscient de la nécessité du contact et des liaisons mécaniques entre la cause et l'effet pour que l'action de la cause ait un caractère de nécessité et d'efficacité, et il prête le pouvoir causal à d'autres personnes ou objets. En somme, il a objectivé et décentré la notion de cause par rapport à lui-même. La pensée adulte décrite par Kant formule le principe de causalité en termes de liaison nécessaire, de dépendance nécessaire de l'effet par rapport à la cause. Elle ajoute à la pensée enfantine de la fin de l'intelligence sensori-motrice le caractère universel du principe de causalité.

L'enfant de 18 mois arrive aussi à coordonner correctement, dans l'ordre voulu, ses séquences d'actions en rapport avec celles des personnes ou objets qui l'entourent. Ceci est encore l'aboutissement d'un long processus de décentration et d'objectivation. Il peut même, à 2 ans, se représenter et situer correctement les uns par rapport aux autres des événements, perçus ou non, qui appartiennent à un passé récent. C'est donc un temps objectif et unique. Celui dont parle Kant est en plus infini et uniforme, ce qui ne révèle pas, du moins pas d'une façon explicite, l'examen de l'intelligence sensori-motrice à son stade final.

Il est donc évident que ce qui constitue un point d'arrivée dans l'étude de Piaget sur la pensée enfantine, constitue une partie du point de départ de Kant dans la Critique de la Raison Pure. Ceci montre clairement que la perspective génétique piagétienne est totalement absente de la pensée kantienne. Le seul cas d'étude "génétique » que connaît Kant est celle de Locke, et il en reconnaît la valeur, tout en affirmant que lui-même fait une toute autre recherche, une recherche sur les conditions qui rendent possible le processus de genèse que décrit Locke :

Les impressions des sens nous fournissent, en effet, la première occasion de déployer à leur sujet toute notre faculté de connaissance et de constituer l'expérience, qui renferme deux éléments très différents : une matière de connaissance fournie par les sens et une certaine forme servant à ordonner cette 
matière et venant de la source intérieure de l'intuition et de la pensée pures, qui ne sont mises en exercice et ne produisent des concepts qu'à l'occasion de la première. Rechercher ainsi les premiers efforts de notre faculté de connaissance pour s'élever des simples perceptions aux concepts généraux, c'est une entreprise qui a sans doute sa très grande utilité, et on doit savoir gré au célèbre LOCKE d'en avoir le premier ouvert la voie. Mais une déduction des concepts purs a priori ne peut jamais être faite de cette manière, car cette voie n'y conduit absolument pas, parce que, relativement à leur usage futur, qui doit être entièrement indépendant de l'expérience, ces concepts doivent présenter un tout autre acte de naissance que celui de leur origine expérimentale. Cette tentative de dérivation physiologique, qui ne peut pas du tout s'appeler proprement déduction, parce qu'elle concerne une question de fait, je l'appellerai donc l'explication de la possession d'une connaissance pure. Il est clair, par suite, qu'il ne peut y avoir de ces concepts d'une déduction transcendantale, et nullement une déduction empirique, et que la dernière, par rapport aux concepts purs à priori n'est qu'une vaine tentative dont peut seul s'occuper celui qui n'a pas saisi la nature tout à fait particulière de ces connaissances ${ }^{12}$.

Pour Piaget, la genèse décrite par Locke est sûrement incomplète, car Locke s'en tient à la seule abstraction simple ou généralisatrice; il ne parle pas de ce que Piaget nomme abstraction réfléchissante, et encore moins de construction de notions. De plus, la genèse dont parle Locke est fort différente de celle que décrit Piaget. La première est abstraction simple à partir d'une expérience ayant un certain contenu, un certain degré de variété et de complexité. Pour Piaget, cette genèse décrite par Locke est elle-même le résultat d'un processus dont les premières phases remontent au début de l'existence: l'abstraction simple et son résultat chez l'adulte (et aussi la construction des notions, dirait Piaget) proviennent par abstraction réfléchissante d'opérations cognitives analogues chez les enfants et les adolescents. L'expérience de ces derniers est moins variée et moins complexe que celle de l'adulte, et ils ont des habitudes mentales différentes, une logique différente (tout au moins dans le cas de l'enfant) et des notions ou prénotions différentes pour interpréter leur expérience. L'évolution progressive, selon des stades déterminés et intégratifs, de cette

12. Critique de la Raison Pure, p. 101. 
expérience, de cette logique, de ces notions et prénotions vers le stade de la pensée adulte est précisément la genèse dont parle Piaget. Il y a conservation de certains aspects des résultats ou représentations antérieures, adjonction de nouveaux éléments et donc transposition des éléments conservés dans une structure représentative plus vaste, plus complexe et autrement structurée, en vue de rendre compte d'une expérience plus riche, plus variée, plus diversifiée et de rendre possible une adaptation efficace par rapport au monde extérieur révélé par cette expérience. C'est en cela précisément que consiste l'abstraction réfléchissante ${ }^{13}$.

\section{L'A PRIORI ET L'INNÉ}

Ceci dit, ni Piaget ni Kant n'identifient l'a priori avec l'inné, et ils ne les considèrent pas non plus comme nécessairement liés. Citons d'abord Piaget :

Quant à l'hérédité de l'intelligence comme telle nous retrouvons la même distinction. D'une part, une question de structure : l' " hérédité spéciale » de l'espèce humaine et de ses "lignées» particulières comporte certains niveaux d'intelligence, supérieurs à celui des singes, etc. Mais d'autre part, l'activité fonctionnelle de la raison (l'ipse intellectus qui ne vient pas de l'expérience) est évidemment liée à l' "hérédité générale " de l'organisation vitale elle-même: de même que l'organisme ne saurait s'adapter aux variations ambiantes s'il n'était pas déjà organisé, de même l'intelligence ne pourrait appréhender aucune donnée extérieure sans certaines fonctions de cohérence (dont le terme ultime est le principe de non-contradiction), de mise en relations, etc, qui sont communes à toute organisation intellectuelle.

Or, ce second type de réalités psychologiques héréditaires est d'une importance capitale pour le développement de l'intelligence. Si vraiment, en effet, il existe un noyau fonctionnel de l'organisation intellectuelle qui procède de l'organisation biologique dans ce qu'elle a de plus général, il est évident que cet invariant orientera l'ensemble des structures successives que la raison va élaborer dans son contact avec le réel : il jouera ainsi le rôle que les philosophes ont attribué à l'a priori, c'est-à-dire qu'il imposera aux structures certaines conditions nécessaires et irréductibles d'existence. Seulement on a eu parfois le tort de

13. Cf. EEG, XIV, p. 203, 217, 223 ; RE, p. 40,395 
regarder l'a priori comme consistant en structures toutes faites et données dès le début du développement, tandis que si l'invariant fonctionnel de la pensée est à l'œuvre dès les stades les plus primitifs, ce n'est que peu à peu qu'il s'impose à la conscience grâce à l'élaboration de structures toujours plus adaptées au fonctionnement lui-même. Dès lors l'a priori ne se présente sous forme de structures nécessaires quau terme de l'évolution des notions et non pas à leur début : tout en étant héréditaire, l'a priori est donc aux antipodes de ce qu'on appelait jadis les «idées innées ».

Quant aux structures du premier type, elles rappellent davantage les idées innées classiques et l'on a pu rajeunir le nativisme à propos de l'espace et des perceptions «bien structurées " du Gestaltisme. Mais, à la différence des invariants d'ordre fonctionnel, ces structures n'ont rien de nécessaire du point de vue de la raison : ce ne sont que des données internes, limitées et limitatives, que l'expérience extérieure et surtout l'activité intellectuelle dépasseront sans cesse. Si elles sont en un sens innées, elles n'ont rien d'a priori, au sens épistémique du terme ${ }^{14}$.

La justice distributive peut être ramenée aux notions d'égalité ou d'équité. Pour l'épistémologie, de tels concepts ne sauraient être qu'a priori si l'on entend par a priori non pas naturellement une idée innée, mais une norme vers laquelle la raison ne peut pas ne pas tendre au fur et à mesure de son épuration. La réciprocité s'impose, en effet, à la raison pratique, comme les principes logiques s'imposent moralement à la raison théorique. Mais, au point de vue psychologique, qui est celui du fait et non plus du droit, une norme a priori n'a d'existence qu'à titre de forme d'équilibre: elle constitue l'équilibre idéal vers lequel tendent les phénomènes, et la question reste entière de savoir, étant donné les faits, pourquoi leur forme d'équilibre est telle plutôt que différente ${ }^{15}$.

Voyons maintenant ce que dit Kant sur le caractère acquis, et non pas inné, des a priori :

La Critique refuse absolument les représentations naturelles ou innées; elle les considere toutes comme acquises, qu'elles appartiennent à l'intuition ou aux concepts de l'entendement.

Mais il existe également (comme disent les professeur de droit naturel) une acquisition originelle, par conséquent aussi de ce qui n'existait pas encore auparavant, de ce qui n'a fait partie

14. NI, p. 9-10

15. JM, p. 253. 
d'aucune chose avant cette action. Telle est, suivant la Critique, premièrement la forme des choses dans l'espace et dans le temps, deuxièmement l'unité synthétique du divers en concepts ; car, aucune des deux n'est tirée des objets comme donnee en eux-mêmes, par notre pouvoir de connaître ; celui-ci y arrive de lui-même a priori. Il doit cependant y avoir dans le sujet un principe qui fait que les représentations conçues naissent ainsi et non autrement et, de plus, qu'elles peuvent se rapporter à des objets qui ne sont pas encore données; ce principe, du moins, est inné ${ }^{16}$.

Le fondement de la possibilité de l'intuition sensible n'est ni image, ni limite du pouvoir de connaître; c'est la simple réceptivité propre à l'esprit lorsqu'il est affecté de quelque chose (dans la sensation), sa qualité de recevoir une représentation conforme à sa constitution subjective. Le premier fondement formel de la possibilité d'une intuition, spatiale par exemple, est seul inné, et non la représentation spatiale elle-même. Toujours, en effet, il faut des impressions pour éveiller notre pouvoir de connaitre et déterminer avant tout la représentation d'un objet (représentation qui, dans tous les cas, est une action propre). Ainsi naît l'intuition formelle qu'on nomme l'espace, comme représentation originairement acquise (représentation de la forme des objets extérieurs en général), dont le fondement (comme simple réceptivité) est cependant inné et dont l'acquisition précède de longtemps le concept déterminé de choses qui s'accordent à cette forme ; l'acquisition de la représentation de ces choses est acquisitio derivativa, étant donné qu'elle suppose déjà des concepts généraux, de caractère transcendantal, de l'entendement, concepts qui ne sont pas non plus innés, mais acquis; cette acquisition, comme celle de l'espace, est originaria et ne suppose d'inné que les conditions subjectives de la spontanéité de la pensée (conformité à l'unité de l'aperception ${ }^{17}$.

L'a priori n'est donc pas nécessairement inné pour Kant. Les seules entités innées qu'il admet sont des lois qui gouvernent la manière dont la sensibilité coordonne les sensations, et l'entendement les phénomènes. Une réflexion sur notre mode de connaître nous révèle, selon lui, des façons constantes de procéder de la sensibilité et de l'entendement, et

16. Réponse à Eberbard, trad. Roger Kempf, Vrin, 1959, p. 71.

17. Ibid., p. 72-73. Voir aussi Dissertation de 1770, trad. Paul Mony, 36 édition, Vrin, 1967 , p. 37, 45, 61, 75: Kant y parle, comme scules réalités innées, de lois de la sensibilité par lesquelles cette dernière coordonne les sensations, et à partir desquelles les notions d'espace et de temps sont abstraites, au cours d'une réflexion de l'esprit sur son mode de connaitre. 
nous abstrayons de ces façons de procéder les notions pures et a priori d'espace, de temps, de substance, de cause, etc.

L'innéisme kantien n'est pas un innéisme cognitif, c'est-àdire que les entités reconnues comme innées ne sont pas des contenus de connaissance, mais seulement des instruments permettant l'acquisition de la connaissance. On peut parler ici d'innéisme instrumental. Piaget défend lui aussi l'innéisme instrumental quand il affirme que la structure sensori-motrice, particulièrement le cerveau, produite par l'évolution biologique et transmise par les parents, est une condition essentielle, bien que non suffisante, de la mise en marche du processus d'apprentissage, condition qui n'est pas elle-même un contenu de connaissance ${ }^{18}$. D'autre part, les entités que Piaget appelle « structures du premier type » dans le texte cité ci-haut, c'est-à-dire par exemple les formes des gestaltistes ou la structure du groupe spatial, sont innées en ce sens qu'elles découlent de la rencontre de l'intelligence avec la réalité extérieure, rencontre où la première fonctionne spontanément d'une manière conforme aux conditions neuro-biologiques mentionnées plus haut. Mais elles ne sont pas pures et a priori au sens kantien de ces termes : elles doivent quelque chose à l'expérience, elles peuvent ne pas être applicables à certains contextes ou à certains types de faits, elles sont susceptibles d'être nuancées ou modifiées pour être applicables, et elles ne sont ni universellement nécessaires ni absolues. Il faut relativiser ici le sens du terme a priori si on veut absolument leur décerner cette étiquette. La même chose vaut à plus forte raison pour l'acquis culturel hérité des générations antérieures : religions, mythes, systèmes philosophiques, théories scientifiques, etc., ne sont pas innés et ne peuvent être qualifiés d'a priori que dans le sens très relatif tout juste mentionné.

L'innéisme instrumental de Piaget et celui de Kant peuvent-ils être identifiés? En d'autres termes, les lois innées dont parle Kant pourraient-elles n'être que l'expression du

18. NI, p. 9. Ce texte a été cité plus haut. Voir aussi BC, p. 38 : "Les processus congitifs apparaissent alors simultanément comme la résultante de l'autorégulation organique dont ils reflètent les mécanismes essentiels et comme les organes les plus différenciés de cette régulation au sein des interactions avec l'extérieur, de telle sorte qu'ils finissent avec l'homme par étendre celles-ci à l'univers. "Voir aussi PE, p. 61-62. 
fonctionnement spontané de la structure sensori-motrice et neurologique et donc être ultimement de nature biologique? Kant n’a ni soutenu ni rejeté une telle hypothèse, et il ne semble pas y avoir songé, bien que rien dans sa pensée ne soit incompatible avec elle, à notre connaissance. De toute façon, l'hypothèse reste discutable, et elle nous semble aller plus loin que Piaget ne le voudrait. Car c'est une chose d'affirmer que la structure sensori-motrice est innée, donnée au départ de la vie cognitive comme condition nécessaire de cette dernière ; mais c'en est une autre de dire que la manière dont nous coordonnons les sensations et les phénomènes ne dépend que de cette structure sensori-motrice, à l'exclusion de tout autre facteur ou condition. Pour Kant, la coordination spontanée, et non apprise, des sensations et des phénomènes consiste à voir les choses comme spatialement juxtaposées selon trois dimensions et comme successives selon une dimension unique et unidirectionnelle, comme fondamentalement stables sous le changement et dépendantes pour leur apparition même, leur existence ou leur devenir, de celle qui précède chacune, etc. Ces comportements cognitifs, à supposer qu'ils soient spontanés plutôt qu'acquis, peuvent aussi être dus, au moins en partie, à l'impact des stimuli dont le monde extérieur bombarde continuellement la structure sensori-motrice. Et cette dernière hypothèse n'implique pas nécessairement une conception très strictement représentative-réaliste de la connaissance.

\section{CONCLUSION}

Si les pensées de Kant et de Piaget sont opposées, ce n'est pas parce que le premier aurait soutenu, contre le second, un innéisme cognitif. Chacun n'affirme qu'un innéisme instrumental dont la nature n'est cependant pas la même dans les deux cas. Plus profondément, l'opposition entre les deux consiste d'abord en ce qu'ils poursuivent des objectifs fort différents. Kant veut expliquer ce qui rend possibles les jugements synthétiques a priori, comme par exemple le principe de causalité, le principe d'égalité de l'action et de la réaction en mécanique et le principe de la permanence de la quantité de matière sous les changements physioco-chimiques, 
qu'il considérait comme absolument universels et nécessaires, et non comme hypothétiques et provisoires ${ }^{19}$. Ceci cautionne, moyennant certaines nuances, l'interprétation de Popper : la physique de Newton, tout au moins en certaines de ses parties et en ses principes fondamentaux, est pour Kant une "épistémè ", c'est-à-dire une connaissance vraie et certaine ${ }^{20}$.

Piaget veut montrer que la genèse des concepts empiriques, telle qu'elle se fait à l'âge adulte, est précédée par d'autres genèses qui aboutissent à des concepts ou préconcepts différents. En d'autres termes il y a une genèse des genèses successives de concepts, et alors que la première est nettement diachronique, on peut à toutes fins pratiques considérer les secondes comme synchroniques. Ce faisant, Piaget s'installe dans une situation où tout contenu de connaissance est contingent et relatif aux limites d'un certain niveau d'expérience plus ou moins élargie, plus ou moins raffinée. La nécessité et l'universalité absolues d'un énoncé ne peuvent être ici que le fruit d'une décision arbitraire ou d'une évaluation naive des conditions de la connaissance. Dans cette perspective, l'existence de concepts purs et de jugements synthétiques a priori n'a aucun sens. Seule la logique formelle encadre les contenus de connaissance.

La problématique kantienne peut certes être contestée. Mais c'est à partir de l'épistémologie moderne des sciences et de l'histoire des sciences depuis l'époque de Kant qu'il faut le faire, et non à partir de l'œuvre de Piaget. Le kantisme ne contient aucune opposition de principe à une ontogenèse de la connaissance. Même si Kant ne parle jamais de l'histoire, du développement ou du progrès de la science, nous ne croyons pas que sa problématique les rende impossibles, mais à la condition qu'ils restent contenus dans les limites définies par des catégories kantiennes. C'est précisément les catégories kantiennes que font éclater la physique relativiste, la mécanique quantique et les géométries non euclidiennes. Ici encore, la logique formelle semble constituer le seul cadre stable. II

19. Critique de la Raison Pure, p. 32-43.

20. Karl R. Popper, Conjectures and Refutations, Harper \& Row, 1968, p. 180, note 17, 185,190 . 
reste à savoir si la structure sensori-motrice est l'unique fondement de cette logique.

Département de Philosophie

Université de Sherbrooke 